\title{
Sacral Insufficiency Fractures Mimicking Lumbar Spine Pathology
}

\author{
G. Sudhir, Kalra K. L., Shankar Acharya, Rupinder Chahal \\ Ortho Spine Department, Sir GangaRam Hospital, New Delhi, India
}

Sacral insufficiency fractures (SIFs) are a common cause of back pain in the elderly. SIFs mimic the symptoms of lumbar spine pathology and so are commonly missed or underdiagnosed. Here we present four cases of missed SIFs that were subsequently identified and treated. One patient was treated as mechanical lower back ache, another patient underwent root block and two patients underwent surgery for lumbar canal stenosis. None experienced relief of their symptoms after these procedures. Retrospective analysis of $X$-ray and magnetic resonance imaging data revealed SIFs that were confirmed by computed tomography scans. All four patients were treated for underlying osteoporosis. Two patients who underwent surgery were treated conservatively and other two were treated by sacroplasty involving injection of cement into the fracture. Sacroplasty produced immediate pain relief and early mobilization compared to the conservative group. SIFs should always be considered in the differential diagnosis of an elderly patient presenting with low back symptoms. Sacroplasty can be considered for immediate pain relief and rapid mobilization.

Keywords: Sacral insufficiency fractures; Osteoporosis; Lumbar pathology

\section{Introduction}

\section{Case Reports}

Sacral insufficiency fractures (SIFs) are a common cause of debilitating back pain in the elderly. SIFs occur in the sacral ala [1]. They tend to occur in the sixth and seventh decades of life, with other risk factors including osteoporosis, multiple myeloma, and Paget's disease [2,3]. The symptoms mimic lumbar spine pathologies like stenosis or metastases, and so SIFs are commonly missed or underdiagnosed. Patients can present with vague back ache with radiculopathy or even with neurological deficit $[4,5]$. These fractures are usually missed in the X-rays and can lead to unnecessary investigations and delayed treatment [6].

Here we present four cases of missed SIFs identified and treated subsequently.

\section{Case 1}

A 78-year-old female with no medical comorbidities presented to with complaints of a 4-month story of low back pain radiating to both lower limbs. She had been treated with antituberculosis treatment (ATT) and analgesics for 1 month prior to presentation. There was no trauma, fever or other joint pain. Back pain was more intense than the leg pain. There was a history of neurogenic claudication. On examination, she had grade 4 power in both lower limbs with normal sensation. Ankle reflexes were diminished with flexor plantars. X-ray examination revealed degenerative changes with osteoporosis, loss of lumbar lordosis with loss of disc space and degenerative

Received Sep 2, 2015; Revised Sep 27, 2015; Accepted Oct 1, 2015

Corresponding author: G. Sudhir

Ortho Spine Department, Sir GangaRam Hospital, Old Rajinder Nagar, New Delhi-110060, India

Tel: +91-99-6227-7126, Fax: +91-011-4225-1947, E-mail: sudhir_axon@yahoo.co.in 
scoliosis from L3-S1. Magnetic resonance imaging (MRI) showed degenerative disc disease from L3-S1 with end plate changes of L3-S1 vertebrae and severe lumbar canal stenosis at L3-4, L4-5, L5-S1 (Fig. 1). She underwent posterior instrumentation with pedicle screws from L3-S1 with posterior midline decompression from L3-S1. Postoperatively, the patient continued to have back pain that prevented walking, even with support. Preoperative neurology was maintained. Postoperative X-ray examination revealed Sacral fracture in the ala and pubic symphysis diastases (Fig. 2). Retrospective analysis of the preoperative MRI revealed edema in the sacral region that had been mistaken as degenerative changes. Bed rest for 3 months along with antiosteoporosis treatment was advised. After 3 months, normal activities.

\section{Case 2}

A 50-year-old, healthy male presented with a 6-month complaint of low back pain radiating to both lower limbs, which was more severe on the left side. Pain was aggravated by activities and was relieved by rest. He was on analgesics for 4 months but had no relief of pain. On examination, tenderness over the lower lumbar spine with restricted extension was evident. Neurology was normal except for diminished ankle reflex on the left side. X-ray examination showed decreased disc space at L4-5 and L5-S1 with narrowing of the foramen and grade 1 listhesis at L4-5. MRI was suggestive of degenerative disc disease at L4-5, L5-S1 with stenosis more on the left side (Fig. 3). Posterior pedicle screw instrumentation L4-S1 with transforaminal lumbar interbody fusion L4-5 and L5-S1 with bone grafts was done (Fig. 4). Postoperatively, the patient was mobile but complained of mild tenderness over the sacral region. Postoperative X-ray examination revealed the presence of SIF in the sacral ala. Bed rest with minimal movement was advised. The patient resumed normal activities. An X-ray at that time revealed good healing of the fracture.

\section{Case 3}

A 30-year-old male with thalessemia major presented with a 5-month history of low back ache radiating to the buttocks. There was no weakness, numbness or history of

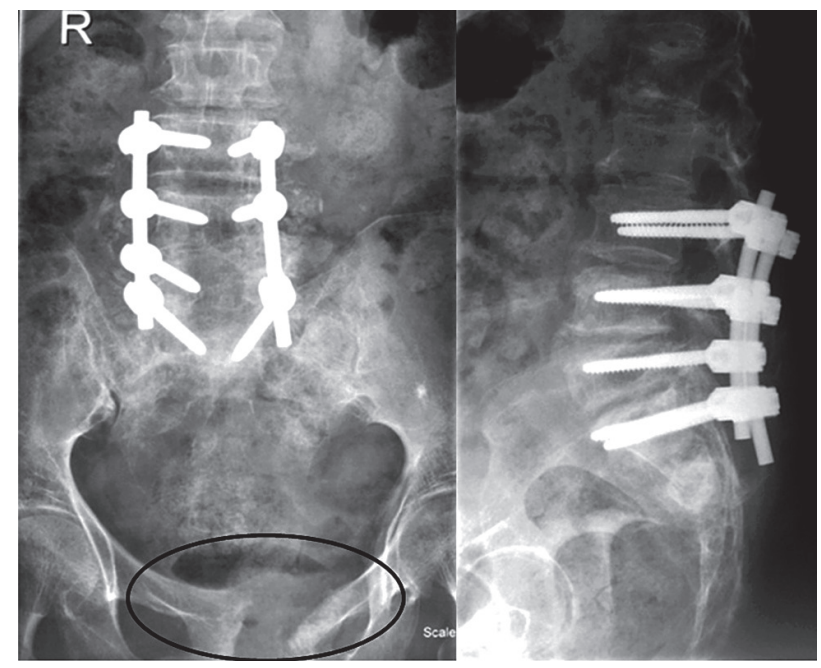

Fig. 2. Postoperative $X$-ray of case 1 showing posterior instrumentation with pubic diastasis probably because of intraoperative manipulation.

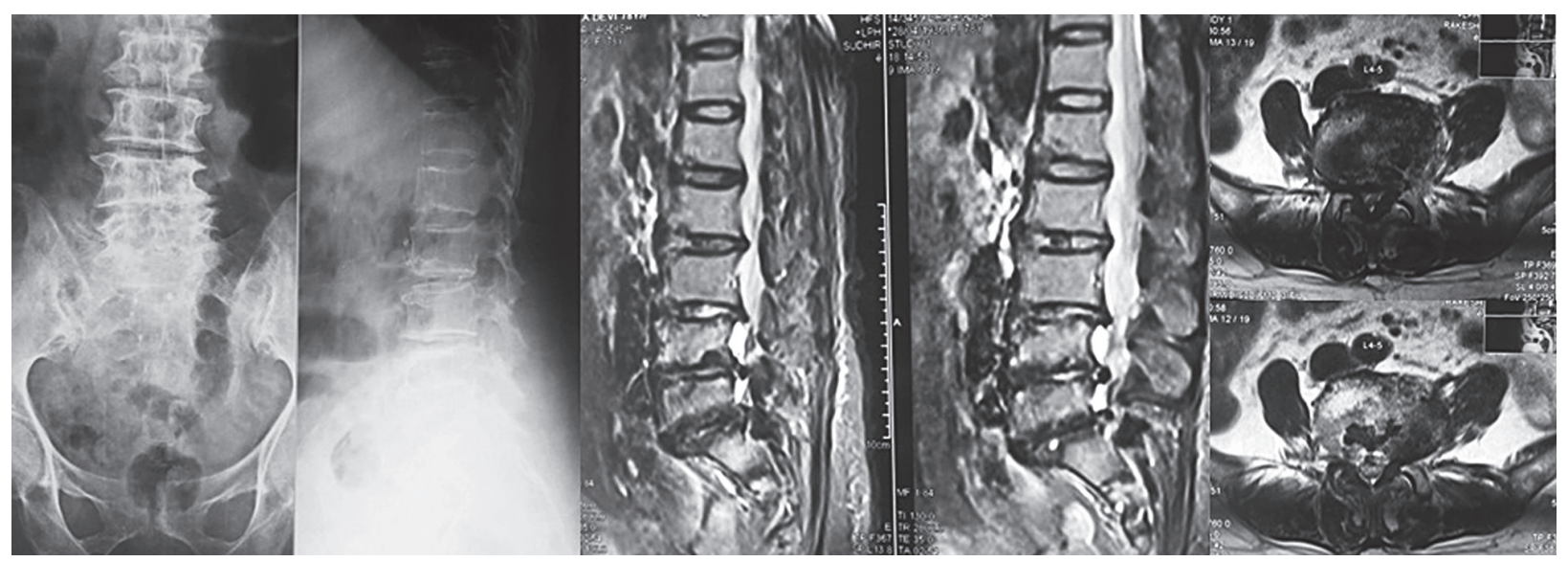

Fig. 1. Preoperative X-ray and magnetic resonance imaging of case 1 showing lumbar canal stenosis at multiple levels with degenerative scoliosis. 


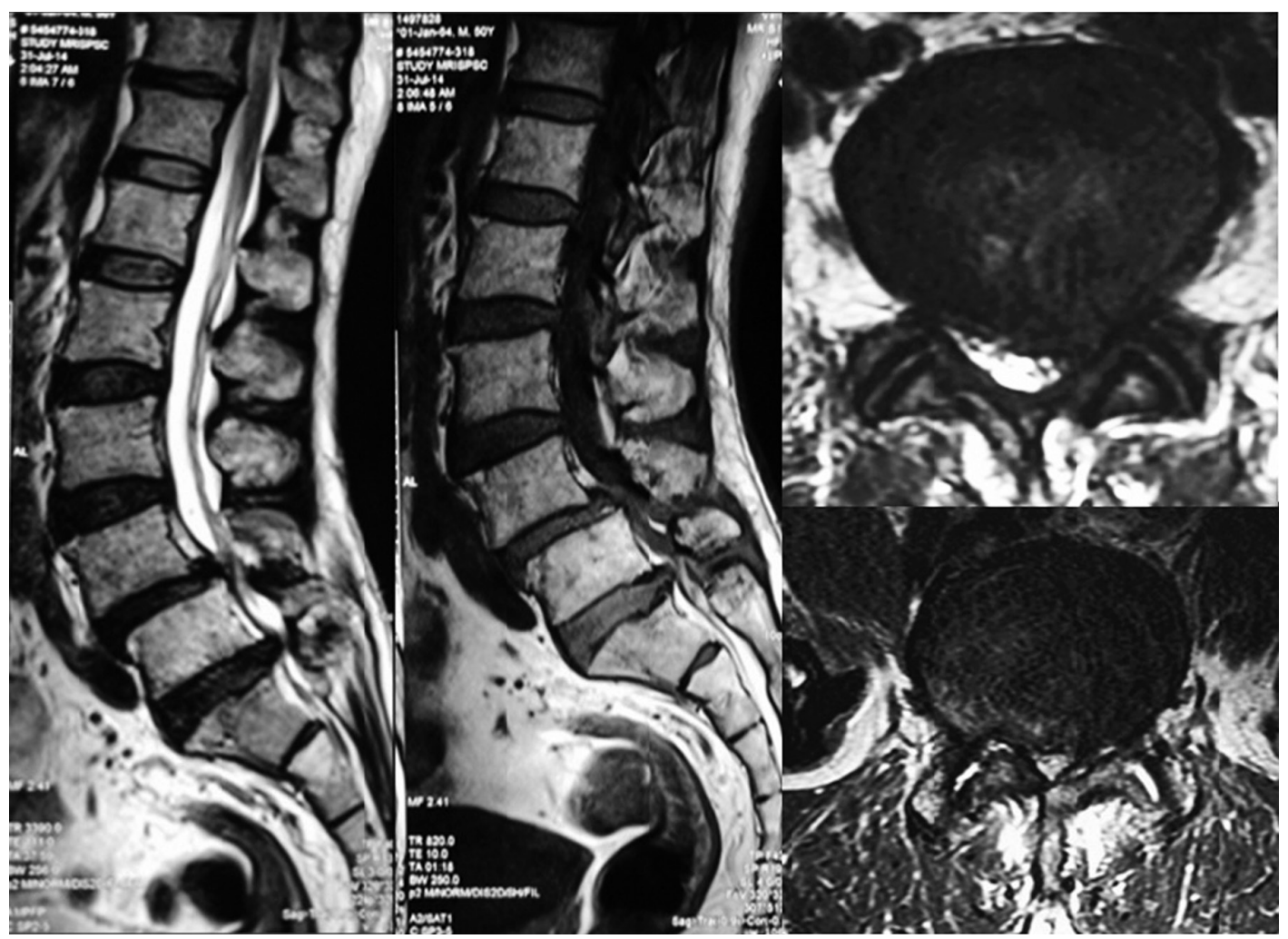

Fig. 3. Preoperative magnetic resonance imaging of case 2 showing lumbar canal stenosis L4-5 and L5-S1 with Grade 1 listhesis at $L 4-5$.

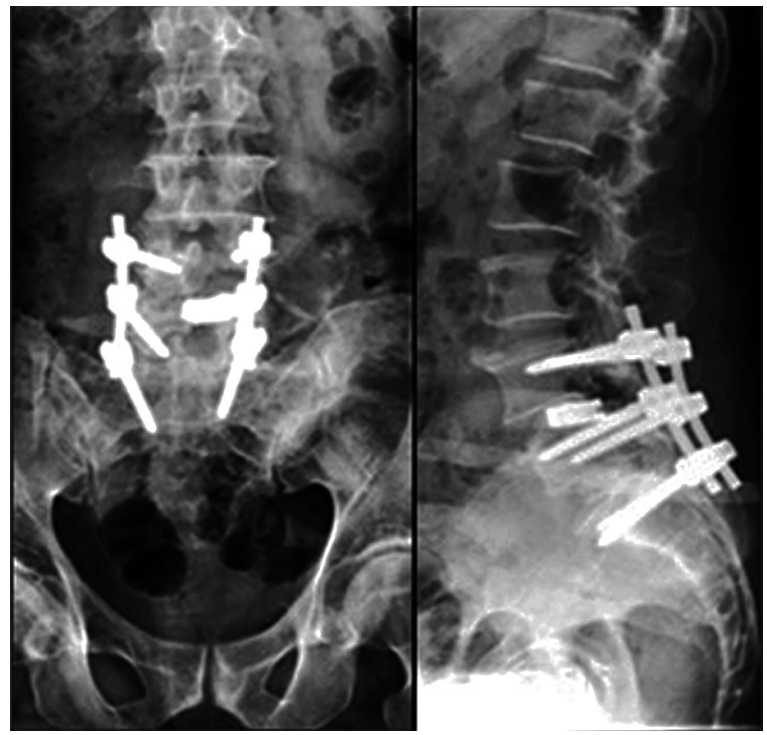

Fig. 4. Postoperative X-ray of Case no. 2 showing posterior instrumentation with transforaminal lumbar interbody fusion cage at L4-5.

trauma. On examination, tenderness in the sacral region with normal neurology was evident. X-ray was normal. MRI revealed hyperintensity in the sacral area with fo- raminal stenosis at the L4-5 region. He received a rightsided L4-5 root block. This did not relieve his pain. A computed tomography (CT) myelogram revealed a SIF with iron deposits in the S1, S2 canal area (Fig. 5). The patient underwent sacroplasty procedure [7] by placing the needle parallel to the sacro iliac joint in the mediolateral direction and parallel to the L5-S1 disc in the craniocaudal direction, and injecting cement into the fracture site bilaterally. The operative time was 20 minutes with negligible blood loss (Figs. 6, 7). The postoperative period was uneventful. The patient was mobilized without any support and discharged on the same day, and resumed normal activities beginning on the second postoperative day.

\section{Case 4}

A 62-year-old, healthy male presented with complaints of low back pain after a trivial fall. Neurology was normal. He had severe tenderness over the sacral region with an antalgic gait. The initial X-ray was unremarkable. He was treated elsewhere with bed rest and analgesics, which brought no relief. MRI was advised. The scan revealed 

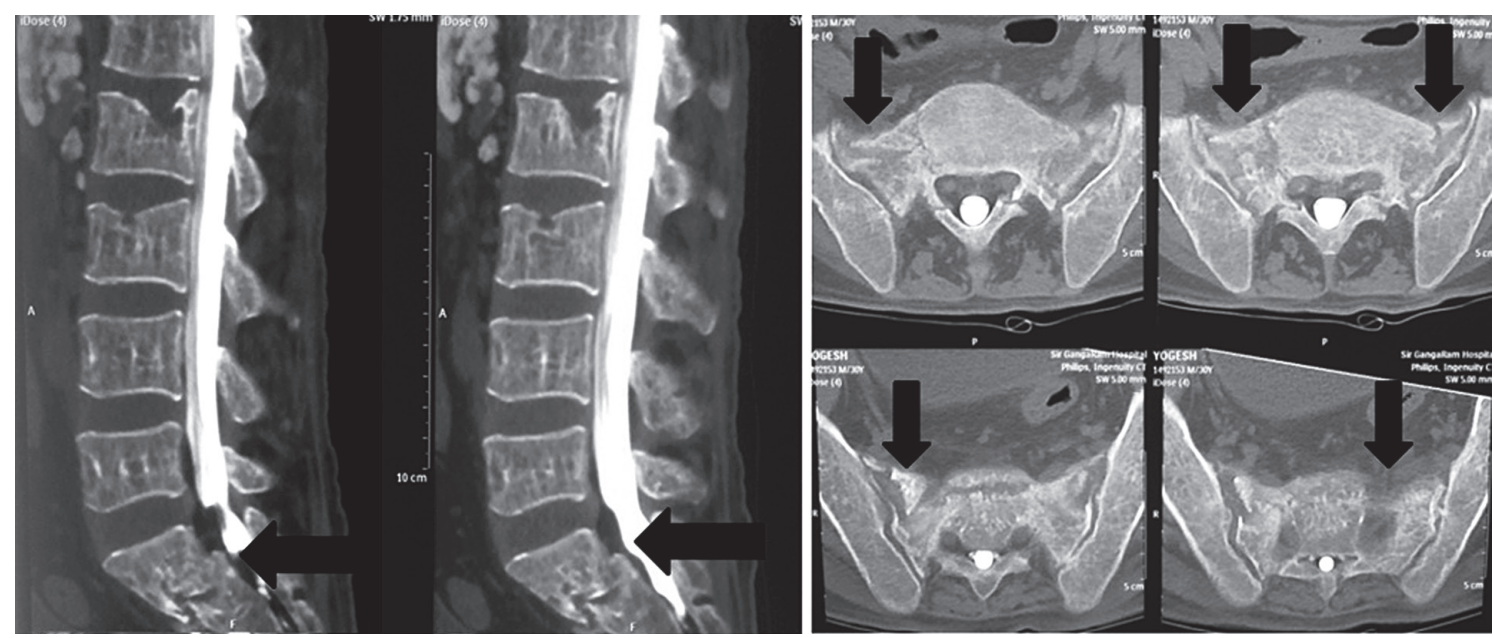

Fig. 5. Preoperative computed tomography scan sagittal and axial views of case 3 showing sacral insufficiency fractures in the sacral ala.

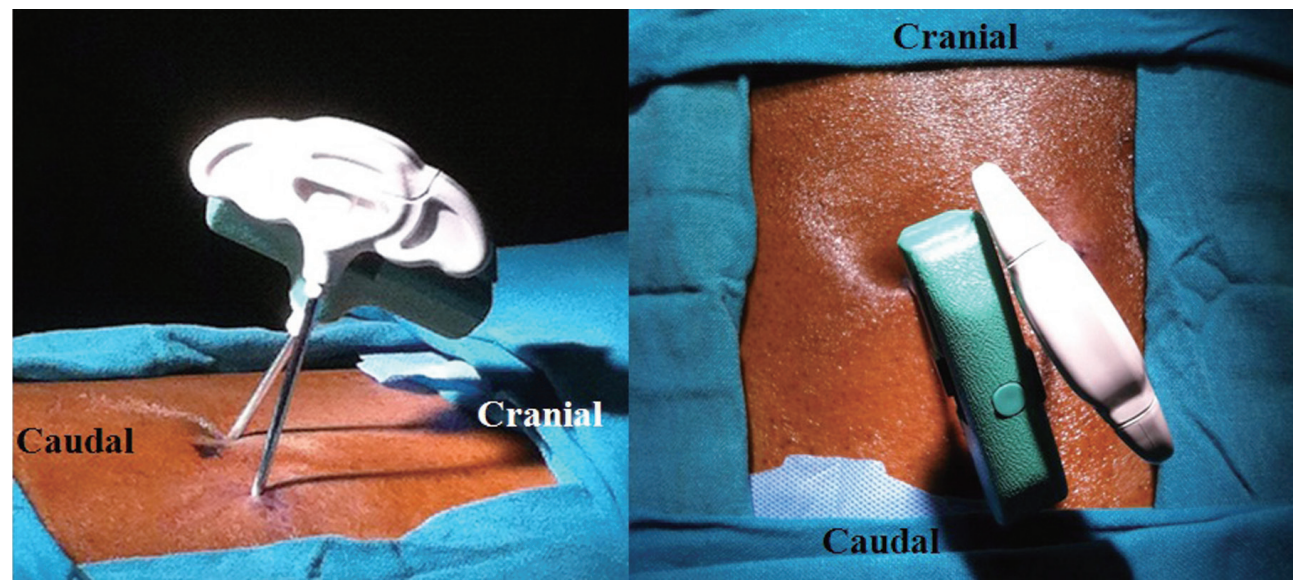

Fig. 6. Intraoperative image of case 3 showing the position of needles in the mediolateral and craniocaudal directions.
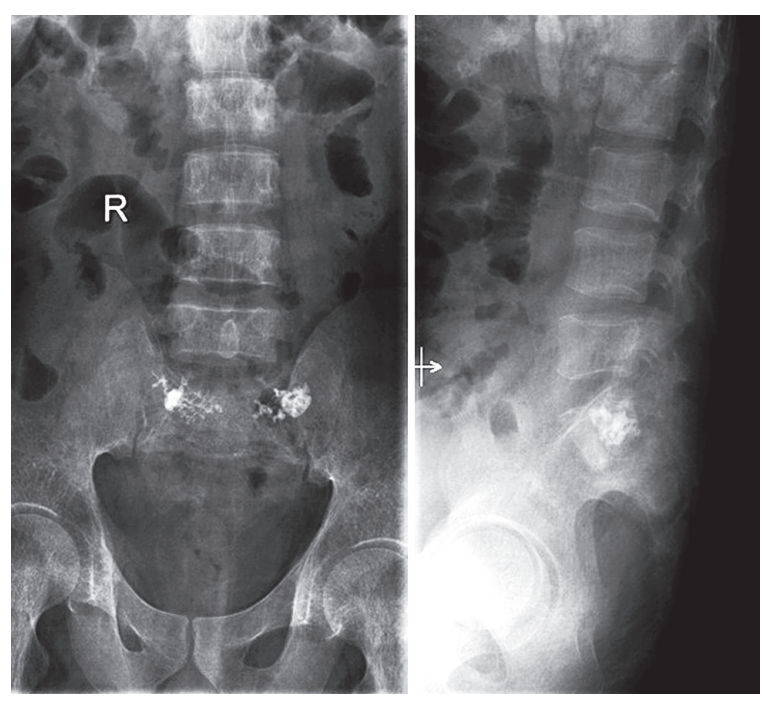

Fig. 7. Postoperative X-ray of case 3 showing cement in the fracture site after sacroplasty. The patient was mobilized and discharged on the same day.

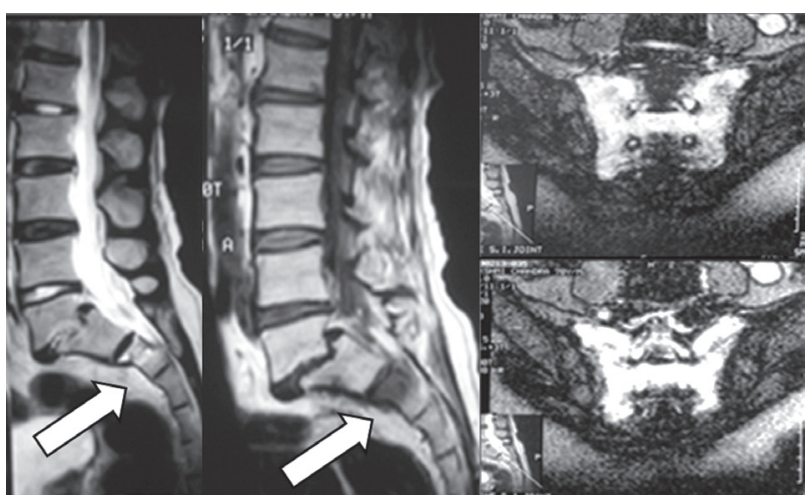

Fig. 8. Preoperative magnetic resonance imaging of Case 4 showing marrow edema (arrow) and $\mathrm{H}$ pattern suggestive of Sacral Insufficieny fracture.

marrow edema in both sacral ala (H Pattern), which was suggestive of SIF (Fig. 8). Since pain was severe even at rest, sacroplasty was advised and was performed as before 

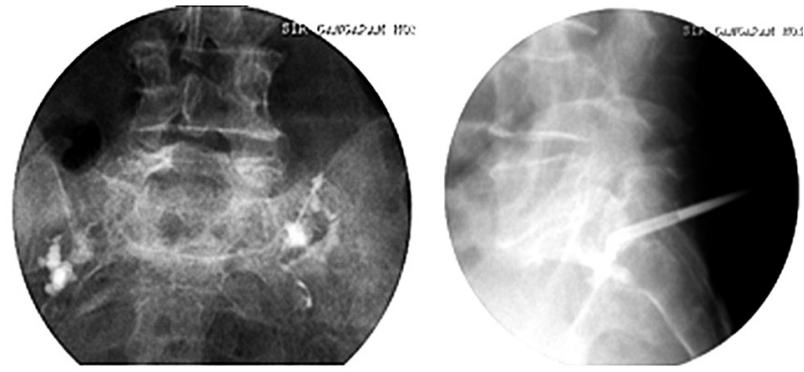

Fig. 9. C-arm image of case 4. Cement in the fracture site after sacroplasty is evident. The patient was mobilized and discharged on the same day.

(Fig. 9). The patient had immediate pain relief and was mobilized and discharged the same day.

\section{Discussion}

Insufficiency fractures are a type of stress fracture that result from normal stress applied to abnormal bone. SIFs were first described by Lourie [8]. They are a common cause of debilitating back pain in the elderly. The incidence of SIFs has been reported as $1 \%$ to $5 \%[9,10]$ in elderly who are at risk. Elderly postmenopausal females with osteoporosis are more prone to develop SIFs [1]. SIF can occur both in males and females over 60 years of age, and is very common between 70 and 75 years of age $[11,12]$. The risk factors include rheumatoid arthritis, Paget disease, radiation, glucocorticoid-induced osteopenia, multiple myeloma, and hyperparathyroidism [2,3]. SIFs are invariably accompanied by severe osteopenia or osteoporosis as measured by dual-energy $\mathrm{X}$-ray absorptiometry. These fractures occur in the sacral ala lateral to the neural foramina and medial to the sacroiliac joints (Dennis zone 1) [13]. Since the sacral ala is composed predominantly of cancellous bone, which is the first to be reduced in cases of osteoporosis and other metabolic causes, it remains the most common region of SIFs. Both unilateral and bilateral presentation can be seen in these fractures; they can also occur in association with pelvic insufficiency fractures, pubic ramii, or acetabulum [14].

Though it occurs without any history of trauma, some patients may present with a history of trivial fall on the buttocks. Patients with SIFs usually have vague symptoms mimicking lumbar spine pathology like lumbar canal stenosis or metastases. Hence, it is missed or underdiagnosed in most circumstances. Patients present with vague low back ache only or with radiating pain to the buttocks
[4], but generally do not present with radiculopathy. However, one case of cauda equina syndrome due to SIF has been described [5]. A metastatic disease-like presentation can also occur resulting in extensive work-up and investigations. In contrast, $45 \%$ of SIFs are associated with malignancy [2]. The pain is usually aggravated by axial loading and by doing activities.

In most instances, X-ray of the lumbosacral spine and pelvis with hips is ordered as these fractures are rarely suspected. The result can be a delay of about 40-55 days from symptom onset before imaging of the sacrum [14]. The fractures are visualized in the sacral ala lateral to the foramen in plain X-rays. In neglected cases or in cases of late presentation they appear as areas of sclerosis [6]. Gotis-Graham et al. [2] reported sclerosis in $57 \%$ of patients, with a clear fracture line seen in only $12.5 \%$ and suggested that only $20 \%$ to $38 \%$ of SIFs can be detected in plain radiographs. Radiographically, SIFs can mimic metastatic lesions with periosteal reaction, which often leads to confusion for the treating physicians [15]. One of the most sensitive tests to detect these fractures is bone scintigraphy with technetium Tc99-labeled methylene diphosphonate [16], which has $96 \%$ sensitivity and $92 \%$ positive predictive value for detecting these fractures. "Honda" or " $\mathrm{H}$ " sign in bone scan is diagnostic of SIFs [17]. Posterior planar images with the sacrum closer to the detector are very sensitive to diagnose these fractures [18]. CT can assist in the diagnosis. Diagnosis is often made from the axial images as these fractures are sagittally oriented. CT sensitivity is $60 \%$ to $75 \%$ [19] and aids in differentiating SIFs and metastases. MRI has a high sensitivity value similar to that of bone scan in diagnosing SIFs. It appears as areas of edema in the sacral ala that short inversion time recovery sequences help to diagnose the edema in the early stages. The fracture line may be evident as hypointensity within the area of edema [19].

The standard treatment of these fractures is conservative including bed rest, rehabilitation and analgesics. Suggestions have included strict bed rest with no ambulation supplemented with analgesics [2] and walker- or crutchaided mobilization with analgesics [12]. This restricted mobility should be continued for a minimum of 3 months or until the pain settles, whichever is earlier. Complications associated with prolonged immobilization including chest infections, bed sores, urinary tract infections, disuse atrophy of the muscles, and demineralization of bones are a setback in this conservative form of treatment [20]. 
Most dreadful of these complications is deep vein thrombosis, which can lead to pulmonary embolism. Mortality following these fractures is low, being reported as $14.3 \%$ [20].

In addition to the complications of conservative treatment some patients have persistent pain that restricts daily activities. In the event of failure of conservative treatment, a new minimally invasive technique sacroplasty by injecting polymethyl methacrylate cement into the sacral fracture is being performed with good outcomes [21,22]. The procedure is similar to the vertebroplasty technique commonly performed for osteoporotic compression fractures. The goals of sacroplasty are immediate pain relief, early mobilization, and rehabilitation to prevent the complications of recumbency. The efficacy and safety of this procedure has been reported $[7,23,24]$ but no prospective randomized controlled trials have compared sacroplasty with conservative treatment. The procedure was first described in 2002 [21]. There are various techniques for sacroplasty, which include the posterior, midline, and long axis approaches $[23,24]$. Each has risks and benefits. Jayaraman et al. [7] described a technique for the safe placement of the needle into the sacrum for injecting the cement. They defined the target point to be at the intersection of two lines, the former joining the posterosuperior corner to the anteroinferior corner and the latter is a line connecting the anterosuperior corner to the posteroinferior corner of $\mathrm{S} 1$ body, which is confirmed by fluoroscopic guidance intraoperatively. In the study, 3\% of subjects had this point anterior to the sacrum. The authors recommended confirming the point preoperatively in multiformated images of the CT scan. The needle trajectory in the mediolateral plane is parallel to the SI joint, whereas in the craniocaudal direction it can have three directions: parallel to the L5-S1 disc space, which is commonly used, along the long axis of sacrum, and axial to the patient. The minimally invasive nature of the technique, negligible blood loss, less operative time, immediate pain relief, and mobilization preventing the complications of prolonged immobilization are benefits. Associated complications include cement extravasation into the vessels, foramen, canal, infection, anaphylaxis, and emboli. Two of our patients who underwent sacroplasty had immediate pain relief and were mobilized and discharged on the same day. They returned to their normal activities after 2 weeks and had no complaints. In our series, all the patients were treated for osteoporosis. Two patients were treated conservatively with bed rest, limited mobilization, and analgesics. The other two were treated by sacroplasty. The latter experienced immediate relief of pain and were discharged on the same day without complications.

This study highlights that SIFs are often underdiagnosed or missed, resulting in unnecessary investigations while increasing morbidity. We have not compared the results of conservative treatment versus sacroplasty for SIFs. A randomized control trial will be required to compare these two treatment methods.

SIFs should always be considered in the differential diagnosis of an elderly patient presenting with low back symptoms. Early diagnosis and prompt treatment can prevent the morbidity associated with these fractures. Sacroplasty is an emerging technique to treat these fractures for immediate pain relief and rapid mobilization.

\section{Conflict of Interest}

No potential conflict of interest relevant to this article was reported.

\section{References}

1. De Smet AA, Neff JR. Pubic and sacral insufficiency fractures: clinical course and radiologic findings. AJR Am J Roentgenol 1985;145:601-6.

2. Gotis-Graham I, McGuigan L, Diamond T, et al. Sacral insufficiency fractures in the elderly. J Bone Joint Surg Br 1994;76:882-6.

3. Dasgupta B, Shah N, Brown H, Gordon TE, Tanqueray AB, Mellor JA. Sacral insufficiency fractures: an unsuspected cause of low back pain. Br J Rheumatol 1998;37:789-93.

4. Rawlings CE 3rd, Wilkins RH, Martinez S, Wilkinson RH Jr. Osteoporotic sacral fractures: a clinical study. Neurosurgery 1988;22:72-6.

5. Muthukumar T, Butt SH, Cassar-Pullicino VN, McCall IW. Cauda equina syndrome presentation of sacral insufficiency fractures. Skeletal Radiol 2007; 36:309-13.

6. Cooper KL, Beabout JW, Swee RG. Insufficiency fractures of the sacrum. Radiology 1985;156:15-20.

7. Jayaraman MV, Chang H, Ahn SH. An easily identifiable anatomic landmark for fluoroscopically guided sacroplasty: anatomic description and validation with treatment in 13 patients. AJNR Am J Neuroradiol 
2009;30:1070-3.

8. Lourie H. Spontaneous osteoporotic fracture of the sacrum: an unrecognized syndrome of the elderly. JAMA 1982;248:715-7.

9. Weber M, Hasler P, Gerber H. Insufficiency fractures of the sacrum: twenty cases and review of the literature. Spine (Phila Pa 1976) 1993;18:2507-12.

10. West SG, Troutner JL, Baker MR, Place HM. Sacral insufficiency fractures in rheumatoid arthritis. Spine (Phila Pa 1976) 1994;19:2117-21.

11. Frey ME, Depalma MJ, Cifu DX, Bhagia SM, Carne W, Daitch JS. Percutaneous sacroplasty for osteoporotic sacral insufficiency fractures: a prospective, multicenter, observational pilot study. Spine J 2008;8:36773.

12. Newhouse KE, el-Khoury GY, Buckwalter JA. Occult sacral fractures in osteopenic patients. J Bone Joint Surg Am 1992;74:1472-7.

13. Denis F, Davis S, Comfort T. Sacral fractures: an important problem: retrospective analysis of 236 cases. Clin Orthop Relat Res 1988;227:67-81.

14. Grangier C, Garcia J, Howarth NR, May M, Rossier P. Role of MRI in the diagnosis of insufficiency fractures of the sacrum and acetabular roof. Skeletal Radiol 1997;26:517-24.

15. Blake SP, Connors AM. Sacral insufficiency fracture. Br J Radiol 2004;77:891-6.

16. Ries T. Detection of osteoporotic sacral fractures with radionuclides. Radiology 1983;146:783-5.
17. Balseiro J, Brower AC, Ziessman HA. Scintigraphic diagnosis of sacral fractures. AJR Am J Roentgenol 1987;148:111-3.

18. Peh WC, Khong PL, Yin Y, et al. Imaging of pelvic insufficiency fractures. Radiographics 1996;16:33548.

19. Cabarrus MC, Ambekar A, Lu Y, Link TM. MRI and CT of insufficiency fractures of the pelvis and the proximal femur. AJR Am J Roentgenol 2008;191:9951001.

20. Babayev M, Lachmann E, Nagler W. The controversy surrounding sacral insufficiency fractures: to ambulate or not to ambulate? Am J Phys Med Rehabil 2000;79:404-9.

21. Garant M. Sacroplasty: a new treatment for sacral insufficiency fracture. J Vasc Interv Radiol 2002; 13:1265-7.

22. Heron J, Connell DA, James SL. CT-guided sacroplasty for the treatment of sacral insufficiency fractures. Clin Radiol 2007;62:1094-100.

23. Butler CL, Given CA 2nd, Michel SJ, Tibbs PA. Percutaneous sacroplasty for the treatment of sacral insufficiency fractures. AJR Am J Roentgenol 2005; 184:1956-9.

24. Strub WM, Hoffmann M, Ernst RJ, Bulas RV. Sacroplasty by $\mathrm{CT}$ and fluoroscopic guidance: is the procedure right for your patient? AJNR Am J Neuroradiol 2007;28:38-41. 